\title{
Ultrasonido contrastado con microburbujas para el diagnóstico no invasivo de hepatocarcinoma
}

\author{
Microbubble-contrast ultrasound for the non-invasive diagnosis of hepatocarcinoma
}

\author{
Alfredo Ramírez Gutiérrez de Velasco, * César A Vega López, ${ }^{\ddagger}$ Adrián F Pérez González, \\ Carlos Paredes Manjarrez, ${ }^{\S}$ Óscar Quiroz Castroll
}

\section{Resumen}

El ultrasonido con medio de contraste (USMC) se aprobó en 2016 para lesiones hepáticas y, desde entonces, ha sido el método diagnóstico para el hepatocarcinoma, cuya sensibilidad y especificidad es muy parecida a la tomografía computarizada y a la resonancia magnética. Las microburbujas se administran por vía intravenosa y tienen una eliminación pulmonar, lo que hace que se pueda utilizar en pacientes nefrópatas. Con el USMC, se pueden valorar en tiempo real las distintas fases de la circulación hepática, por lo que el hepatocarcinoma tiene características específicas que, según el LI-RADS (Liver Imaging Reporting and Data System), se puede tener una certeza bastante elevada de su diagnóstico. Se concluye que el USMC tiene ventajas y desventajas para el diagnóstico y seguimiento de pacientes con hepatocarcinoma, por lo que se deberán de tomar en cuenta al momento de estudiar a estos pacientes y considerarla como una herramienta eficaz para el mismo.

Palabras clave: Hepatocarcinoma, ultrasonido contrastado, microburbujas, LI-RADS.

\section{INTRODUCCIÓN}

El ultrasonido con medio de contraste (USMC) inició en 1990, sin embargo, fue hasta 2016 que la Food and Drug Administration (FDA), en Estados Unidos, lo aprobó para su uso en lesiones hepáticas. Desde entonces, este método diagnóstico ha ganado popularidad alrededor del mundo. El USMC es un método que nos ayuda a tener una mejor visualización de las estructuras anatómicas y de las lesiones. Su uso más común es transcutáneo, aunque también

\section{Abstract}

Contrast-Enhanced Ultrasound (CEUS) was approved in 2016 for its use in hepatic lesions. Ever since its approval, it has been used for the diagnosis of Hepatocellular Carcinoma (HCC) with a similar sensitivity and specificity than Computed Tomography and Magnetic Resonance. The microbubles are administered intravenously and have pulmonary elimination, which makes them an ideal study for patients with chronic kidney disease. CEUS allows us to evaluate hepatic lesions in real time with their different contrast phases, and recognize the specific features of HCC that according to the LI-RADS classification (Liver Imaging Reporting and Data System), it can be diagnosed with high accuracy. CEUS has many advantages and disadvantages for the diagnosis and follow up of HCC, they should all be taken into consideration during the evaluation of these patients and consider CEUS as a valuable diagnostic tool.

Keywords: Hepatocarcinoma, contrast ultrasound, microbubbles, LI-RADS.

se puede usar de forma transoperatoria. En la actualidad, existen varios medios de contraste disponibles para el ultrasonido; no obstante, en México sólo está disponible el SonoVue (Bracco).

EI USMC consiste en inyectar por vía intravenosa microburbujas con hexafluoruro de azufre, las cuales tienen un tamaño de 2 a $10 \mu \mathrm{m}$. Para el hígado, se utiliza un transductor tradicional convexo de $3-5 \mathrm{mHz}$, además de un software especial como medio de contraste, que consiste en tener una imagen con un índice mecánico bajo (0.05-
* Residente de Imagenología Diagnóstica y Terapéutica.

₹ Medicina Interna.

§ Médico adscrito al Departamento de Imagenología.

Il Jefe del Departamento de Imagenología.

Hospital Ángeles Pedregal. Ciudad de México, México.
Correspondencia:

Alfredo Ramírez Gutiérrez de Velasco

Correo electrónico: alfredomd@me.com

Aceptado: 05-07-2019. 
0.30). De esta forma, se puede observar la alta reflectividad de las microburbujas al cruzar con el haz del ultrasonido. ${ }^{1}$

A diferencia de los medios de contraste para la tomografía computarizada (TC) y la resonancia magnética (RM), estas microburbujas tienen una distribución puramente intravascular, por lo que se puede visualizar tanto la macro como la microvasculatura. Además, su tamaño permite el paso de las microburbujas a través de la circulación pulmonar, por lo que sólo se necesita una dosis de 2 a $4 \mathrm{~mL}$, debido a la recirculación de las mismas.

EI USMC puede ser utilizado para valorar las lesiones focales del hígado, guiar las biopsias o planear y monitorizar la respuesta al tratamiento de pacientes con tumores hepáticos y se puede emplear para valorar a los pacientes para trasplante hepático; además, tiene una gran habilidad para diferenciar lesiones benignas y malignas en pacientes con parénquima sano y para detectar metástasis hepáticas.

Existen estudios que ponen al USMC a la par de la TC y la RM en el diagnóstico de cáncer hepático; éstos reportan una sensibilidad de 27 a $98 \%$ para USMC, de 47 a $98 \%$ para TC con medio de contraste (MC) y de 44 a $97 \%$ para RM con MC; también se ha reportado una especificidad de 50 a $100 \%$, 71 a $100 \%$ y de 62 a $100 \%$, respectivamente. ${ }^{2}$

\section{CASO CLÍNICO}

Se presentó un hombre de 58 años, con antecedente de diabetes mellitus de varios años de evolución y sin tratamiento actual. Negó antecedentes de alcoholismo o tabaquismo. Acudió al Servicio de Urgencias por presentar un cuadro clínico de seis horas de evolución de evacuaciones melénicas en tres ocasiones con abundante cantidad, motivo por el cual se le realizó una endoscopia de tubo digestivo, que reportó la presencia de varices esofágicas grado III, las cuales ameritaron ligadura. Debido a este hallazgo, se realizó ultrasonido hepático y tomografía contrastada de abdomen, donde se observaron hallazgos sugestivos de hepatocarcinoma. Por ello, se decidió realizar USMC para mejorar la determinación de la lesión.

Se administró por vía intravenosa $2.4 \mathrm{~mL}$ de ecorrealzador (SonoVue ${ }^{\circledR}$ Bracco) y, a su vez, se mantuvo en el campo de visión a la masa hepática, la cual midió $50 \times$ $60 \mathrm{~mm}$ y se contabilizó el tiempo desde el momento de la inyección. A los 25 segundos, se comenzó a ver un realce homogéneo de la masa (Figura 1A) en comparación con el parénquima hepático; posteriormente, a los 76 segundos (Figura 1B), se observó un lavado de las microburbujas. ${ }^{3}$

\section{DISCUSIÓN}

Las microburbujas consisten de un gas inerte con una cobertura lipídica que las estabiliza y tienen una distri- bución intravascular estricta. Es precisamente por esta característica que puede demostrar patrones de lavado de forma más confiable que la TC y la RM. La eliminación de las microburbujas es por vía pulmonar, por lo que no se necesitan pruebas de función renal para realizarse y se puede administrar de manera segura en pacientes nefrópatas. Sus reacciones adversas son muy raras y leves, pues en una serie de 101,571 inyecciones se ha reportado tan sólo una reacción adversa por cada 10,000.3,4

Al igual que los medios de contaste para TC y RM, las microburbujas tienen todas las fases vasculares. La fase arterial comienza de 10 a 20 segundos después de la inyección y dura de 25 a 35 segundos. La portal-venosa inicia unos segundos después (de 30 a 45 segundos después de la inyección) y dura hasta 120 segundos después de la inyección. Posterior a esto, se conoce como fase tardía, y suele durar hasta que el medio de contraste sale de la circulación, lo cual puede durar entre cuatro y seis minutos.
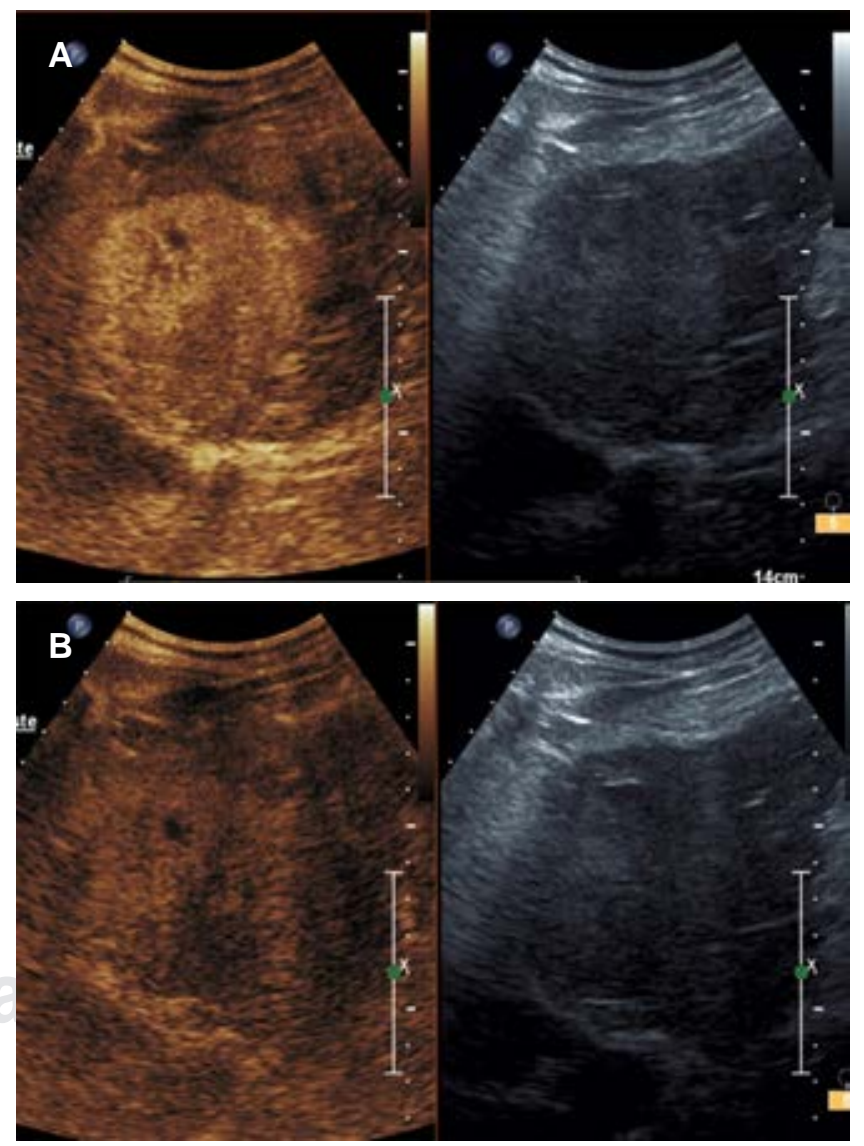

Figura 1: (A) Ultrasonido contrastado con masa hepática con reforzamiento a los 17 segundos de administración de microburbujas. (B) Lavado del medio de contraste de la masa a los 76 segundos. 
Tabla 1: LI-RADS de ultrasonido con medio de contraste (Contrast Enhanced Ultrasound LI-RADS).

Reforzamiento en fase

arterial
Sin RFA

RFA

\begin{tabular}{|c|c|c|c|c|}
\hline Tamaño del nódulo (mm) & $\begin{array}{l}<20 \\
<<2\end{array}$ & $\geq 20$ & $\begin{array}{l}<10 \\
<3\end{array}$ & $\geq 10$ \\
\hline Sin lavado & CEUS LR-3 & CEUS LR-3 & CEUS LR-3 & CEUS LR-4 \\
\hline
\end{tabular}

LI-RADS = Liver Imaging Reporting and Data System; RFA = reforzamiento en fase arterial.

Debido al doble aporte sanguíneo del hígado por la arteria hepática $(25-30 \%)$ y la vena porta $(70-75 \%)$, es muy importante la valoración de la dinámica del medio de contraste en lesiones hepáticas, especialmente en la fase de reforzamiento arterial y lavado, ${ }^{5}$ las cuales nos permiten realizar un diagnóstico certero, ya que la neoangiogénesis de los tumores malignos del hígado permite un reforzamiento en la fase arterial sin importar su grado de diferenciación. ${ }^{6} \mathrm{El}$ ultrasonido, a diferencia de la TC y la RM, realiza una valoración completa y en tiempo real de todas las fases hepáticas, y es mas consistente para ver patrones de reforzamiento y lavado tempranos, los cuales pueden perderse en las otras modalidades debido a la biodistribución de los medios de contraste, como la extravasación al intersticio y los factores de distribución dependientes del paciente. ${ }^{7}$

En 2011, el Colegio Americano de Radiología (ACR, por sus siglas en inglés) publicó la primera versión del LIRADS (Liver Imaging Reporting and Data System), la cual sirve como un sistema estandarizado para la terminología y criterios que los radiólogos deben utilizar para interpretar y reportar los estudios del hígado en pacientes con riesgo para hepatocarcinoma (HCC).

En sus inicios, el LI-RADS fue dirigido únicamente para la TC y la RM, pero, en su versión de 2014, se agregaron los medios de contraste hepatobiliares de la RM y, en 2016, se incorporó el USMC; ${ }^{8}$ esto debido a que se le considera al USMC como una herramienta eficaz para diferenciar lesiones hepáticas malignas. El CEU LI-RADS (Contrast Enhanced Ultrasound LI-RADS por sus siglas en inglés) tomó en cuenta el tamaño del tumor, su patrón de reforzamiento y el lavado; estas mismas características son las que se utilizan en la valoración por TC y RM (Tabla 1). En este escenario, el USMC tiene una sensibilidad y especificidad de 95 y $94 \%$ respectivamente para TC y RM. ${ }^{9}$

El paciente actual presentó un reforzamiento en la fase arterial de la masa hepática con un lavado tardío. Este hallazgo, sumado al tamaño del tumor, clasifica a la lesión como un CEU LI-RADS 5 (en definitiva como HCC). Al paciente también se le realizó una TC contrastada (Figura 2), obteniendo un puntaje de 4 de acuerdo con LI-RADS (probable HCC), ${ }^{10}$ por lo que podemos concluir que el USMC tuvo en esta ocasión una mejor sensibilidad diagnóstica que la TC.

El USMC es un método económico, portátil y rápido, que implica menos riesgo que los contrastes utilizados en TC y RM. Consideramos que existe evidencia suficiente, actualmente, para que el USMC sea una opción válida en el diagnóstico de masas hepáticas y en pacientes que requieren seguimiento de estas lesiones (Tabla 2). Además tiene especial indicación en pacientes con función renal disminuida, pacientes con lesiones que no pueden ser caracterizadas por otro método, pacientes que no puedan ser trasladados al departamento de Imagenología o en aquéllos que requieran seguimiento periódico para ver cambios posteriores al tratamiento.

\section{CONCLUSIÓN}

EI USMC es una herramienta eficaz para caracterizar lesiones hepáticas. También es una opción confiable para el

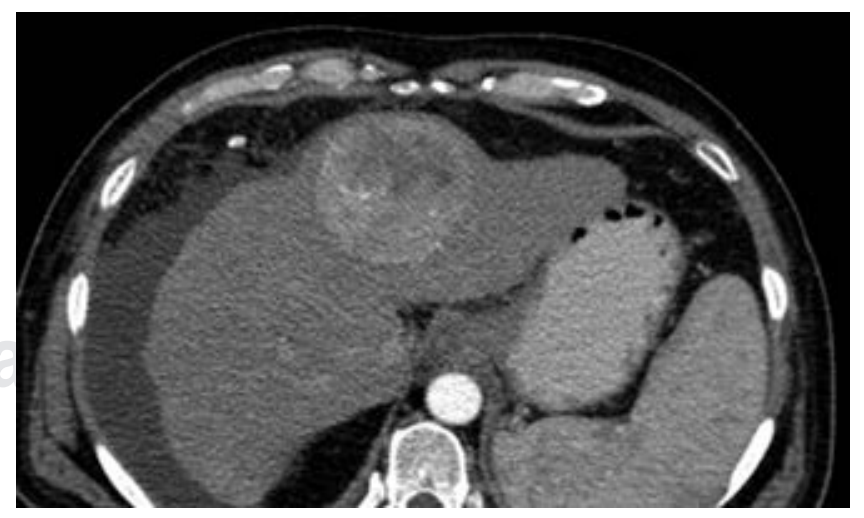

Figura 2: Tomografía computarizada multicorte del abdomen con medio de contraste intravenoso en fase arterial que revela la presencia de una masa hepática en el lóbulo izquierdo de $6 \times 5 \mathrm{~mm}$ con reforzamiento generalizado de la masa. 
Tabla 2: Ventajas y desventajas del ultrasonido con medio de contraste sobre la tomografía computarizada y la radiografía magnética.
Ventajas

- Menor costo

- Alta disponibilidad

- Sin radiación ionizante

- Portátil

- Efectos adversos graves casi nulos

- Eliminación pulmonar

- Se puede realizar en pacientes alérgicos al yodo

- No causa hepatotoxicidad o nefrotoxicidad
Desventajas

- Operador dependiente

- Limitado campo de visión

- No detecta lesiones menores a 3-5 mm

- Penetración hepática limitada

- No todas las lesiones son accesibles para visualizar (segmento 7-8)

- Es difícil diferenciar las lesiones benignas en pacientes cirróticos abordaje y seguimiento de los pacientes con sospecha de HCC, sobre todo en pacientes que tengan contraindicaciones para realizarles ya sea una TC o una RM.

\section{REFERENCIAS}

1. Ferraioli G, Meloni M. Contrast-enhanced ultrasonography of the liver using SonoVue. Ultrasonography. 2018; 37: 25-35.
2. Konopke R, Kersting S, Bergert H. Contrast-enhanced ultrasonography to detect liver metastases : a prospective trial to compare transcutaneous unenhanced and contrast-enhanced ultrasonography in patients undergoing laparotomy. Int J Colorectal Dis. 2007; 22: 201.

3. Dietrich CF, Averkiou M, Nielsen MB, Barr RG, Burns PN, Calliada $F$ et al. How to perform contrasted-enhanced ultrasound (CEUS). Ultasound Int Open. 2017; E2-E15.

4. Piscaglia F, Bolondi L, Italian Society for Ultrasound in Medicine and Biology (SIUMB) Study Group on Ultra-sound Contrast Agents. The safety of Sonovue in abdominal applications: retrospective analysis of 23,188 investigations. Ultrasound Med Bio. 2006; 32 (9): 1369-1375.

5. Wei K, Mulvagh SL, Carson L, Davidoff R, Gabriel R, Grimm RA et al. The safety of DeFinity and Optison for ultrasound image enhancement: a retrospective analysis of 78,383 administered contrast doses. J Am Soc Echocardiogr. 2008; 21 (11): 1202-1206.

6. Claudon M, Dietrich CF, Choi BI. Guidelines and good clinical practice recommendations for Contrast Enhanced Ultrasound (CEUS) in the liver -update 2012: A WFUMB-EFSUMB initiative in cooperation with representatives of AFSUMB, AIUM, ASUM, FLAUS and ICUS. Ultrasound Med Biol. 2013; 39: 187.

7. Liu X, Jang HJ, Khalili K, Kim TK, Atri M. Successful integration of contrast enhanced us into routine abdominal imaging. Radiographics. 2018; 38: 1454-1477.

8. American College of Radiology. ACR CEUS LI-RADS 2017 [Internet]. American College of Radiology. Disponible en: https://www.acr.org/ Clinical-Resources/Reporting-and-Data-Systems/LI-RADS/CEUS-LIRADS-v2017 [accesado el 31 de agosto de 2018].

9. Seitz K, Bernatik T, Strobel D, Blank W, Friedrich-Rust M, Strunk $\mathrm{H}$, Greis $\mathrm{C}$ et al. Contrast-enhanced ultrasound (CEUS) for the characterization of focal liver lesions in clinical practice (DEGUM Multicenter Trial): CEUS vs MRI--a prospective comparison in 269 patients. Ultraschall Med. 2010; 31: 492.

10. Isayes KM, Kielar AZ, Chernyak V, Morshid A, Furlan A, Marks RM et al. LI-RADS: a conceptual and historical review from its beginning to its recent integration into AASLD clinical practice guidance. $J$ Hepatocell Carcinoma. 2019; 6, 46-69. 